\title{
Classical History Theory of Vector Fields
}

\author{
Duncan Noltingk* \\ Blackett Laboratory \\ Imperial College \\ Prince Consort Road \\ London SW7 2BZ
}

November 2, 2018

\begin{abstract}
We consider the extension of classical history theory to the massive vector field and electromagnetism. It is argued that the action of the two Poincare groups introduced by Savvidou suggests that the history fields should have five components. The extra degrees of freedom introduced to make the fields five-dimensional result in an extra pair of second class constraints in the case of the massive vector field, and in an extended gauge group in the case of electromagnetism. The total gauge transformations depend on two arbitrary parameters, and contain 'internal' and 'external' $U(1)$ gauge transformations as subgroups.
\end{abstract}

*e-mail d.noltingk@ic.ac.uk 


\section{Introduction}

\subsection{Motivation}

The Hamiltonian formalism provides a strong link between classical and quantum theories and is mathematically well-developed in both cases. However, a major drawback of the standard Hamiltonian approach to relativistic field theories is that it requires a splitting of space-time into space and time, thus breaking the manifest covariance of the theory. This problem becomes particularly acute when canonical methods are applied to generally covariant theories such as general relativity, and this is one aspect of the "problem of time' in canonical quantum gravity.

Recently a Hamiltonian formalism has been proposed by Isham and coworkers [1], 2, 3], in which the fundamental physical entities are entire histories of the system under consideration, as opposed to Cauchy data at an instant of time. For a thorough introduction see [5]. The histories formalism was originally developed in the quantum case, but there is a well-defined classical history formalism. The central object in a classical history theory is the space of histories, $\Pi$, which is defined to be a one-parameter family of singletime phase spaces. An element of $\Pi$ is called a history, and the space of histories carries a symplectic structure which provides the crucial link to the corresponding quantum history theory.

A particularly striking fact about history theories is that there are two notions of time evolution [6]. External time corresponds to the parameter labeling the copies of state space, and internal time is generated by Hamiltonian evolution. In this way the notion of time plays two different roles in a history theory, one corresponding to the causal ordering of logical propositions, and the second corresponding to dynamical evolution. The two times are linked together in the action principle.

The histories formalism has been applied to scalar field theory on flat [7] and curved [9, 10] space-times, and in the following we discuss the extension of the classical theory to the case of vector fields. In particular we examine the massive vector field and the electromagnetic field from a histories perspective. We shall argue that, as a consequence of the two time directions, a history field should be regarded as a certain type of vector field on a five-dimensional space-time. We also give an analysis of the gauge transformations of histories electromagnetism. 
These results are relevant to the ultimate goal of the histories programme: the formulation of a histories version of general relativity [8]. Firstly, they suggest the possibility that a covariant history theory of gravity should be concerned with the metric of a five-dimensional extended space-time. Secondly, gauge symmetry is an important feature of general relativity, particularly when formulated in terms of tetrad fields. The study of the extended gauge transformations of histories electromagnetism is relevant in this context.

\subsection{Scalar Field Theory}

In this section we give a brief account of the histories description of the classical scalar field on Minkowski space-time, $\mathcal{M}$, as given by Savvidou [7]. We define the Minkowski metric $\eta_{\mu \nu}$ to have signature $(+,-,-,-)$.

\subsubsection{The history algebra}

The canonical configuration space of the scalar field is $Q_{n, t}=C^{\infty}\left(\Sigma_{n, t}\right)$ where $\Sigma_{n, t}$ is a Cauchy surface in $\mathcal{M}$. The Cauchy surfaces are labeled by a future pointing timelike unit vector $n$ normal to $\Sigma_{n, t}$, and a real number $t$. Each Cauchy surface represents an instant of time in a particular inertial frame. The state space $P_{n, t}$ is the cotangent bundle of $Q_{n, t}$ which we identify with $C^{\infty}\left(\Sigma_{n, t}\right) \times C^{\infty}\left(\Sigma_{n, t}\right)$ '. The construction of the corresponding history theory begins by defining a trivial vector bundle fl $\xi_{n}: P \times \mathbb{R} \rightarrow \mathbb{R}$ such that $\xi_{n}^{-1}(t)=P_{n, t}$. Here $P$ is an abstract copy of the state space. The space of histories of the scalar field corresponds to the space of sections of this bundle, $\Pi_{n}=\Gamma\left(\xi_{n}\right)$, and the history fields satisfy the following Poisson algebra:

$$
\begin{aligned}
& \left\{\phi_{n}(t ; \underline{x}), \phi_{n}\left(t^{\prime} ; \underline{x}^{\prime}\right)\right\}=0 \\
& \left\{\pi_{n}(t ; \underline{x}), \pi_{n}\left(t^{\prime} ; \underline{x}^{\prime}\right)\right\}=0 \\
& \left\{\phi_{n}(t ; \underline{x}), \pi_{n}\left(t^{\prime} ; \underline{x^{\prime}}\right)\right\}=\delta\left(t-t^{\prime}\right) \delta_{n, t}^{(3)}\left(\underline{x}-\underline{x^{\prime}}\right)
\end{aligned}
$$

\footnotetext{
${ }^{1}$ The analytical subtleties regarding these infinite dimensional spaces will not concern us here. We shall only be interested in the resulting Poisson algebra.

${ }^{2}$ In this paper we will not make use of this bundle structure, and we could just have defined $\Pi_{n}$ to be the space of paths $\mathbb{R} \rightarrow P_{n}$. However, the bundle picture is useful in more general situations (eg., on curved space-times), and also gives a motivation for the 'internal' / 'external' nomenclature in history theory: internal transformations act internally to the fibres of $\xi_{n}$, while external transformations act across the fibres.
} 
where $\delta_{n, t}^{(3)}\left(\underline{x}-\underline{x^{\prime}}\right)$ is the delta function on $\Sigma_{n, t}$. The right hand side of this equation is a space-time scalar density of weight one. In Minkowski space-time, there is no difference between a scalar density and a scalar (if we restrict attention to transformations under the connected part of the Poincare group), which suggests that the fields can be thought of as spacetime scalars. A pair $(t, \underline{x}) \in \mathbb{R} \times \Sigma$ can be identified with a unique four-vector $X \in \mathcal{M}$ as $X=t n+x_{n}$, where the three-vector $\underline{x}$ has been associated with a corresponding four-vector $x_{n}$ that is $n$-spatial (i.e., $n \cdot x_{n}:=\eta_{\mu \nu} n^{\mu} x_{n}^{\nu}=0$ ). Hence we can write $\phi_{n}(t ; \underline{x})$ as $\phi_{n}(X)$. The history algebra can then be written in the more covariant looking form

$$
\begin{aligned}
& \left\{\phi_{n}(X), \phi_{n}\left(X^{\prime}\right)\right\}=0 \\
& \left\{\pi_{n}(X), \pi_{n}\left(X^{\prime}\right)\right\}=0 \\
& \left\{\phi_{n}(X), \pi_{n}\left(X^{\prime}\right)\right\}=\delta^{(4)}\left(X-X^{\prime}\right)
\end{aligned}
$$

Now it is tempting to drop the $n$ label from the fields since the right hand side of this algebra does not depend on $n$. However, this would be somewhat misleading in the sense that the field $\pi(X)$ has no physical meaning. This is because the conjugate momentum corresponds to the field momentum along a certain timelike direction, and so must be written as $\pi_{n}(X)$. Although the algebra (4), (5), (6) is independent of $n$, Savvidou [7] has shown that in the quantum theory $n$ labels the physically relevant, inequivalent representations of the algebra on a particular Fock space. So in the analysis of the classical theory the $n$-labels remain on the fields on the understanding that they are necessary for the physical interpretation of the theory and arise naturally in quantisation. However, we note that it is rather unsatisfactory to have an $n$ label on the $\phi$ field because, physically, the value of the field at a point in space-time is independent of the foliation. In this histories formulation of classical scalar field theory all propositions about the field are made in the context of a particular inertial reference frame ${ }^{3}$.

\subsubsection{Time translations}

For each $n$, a 'Louville' operator can be constructed from the fields,

$$
V_{n}:=\int d^{4} X \pi_{n} n_{\mu} \partial^{\mu} \phi_{n}
$$

\footnotetext{
${ }^{3}$ The 'multisymplectic' approach to field theory offers a way round this problem as it

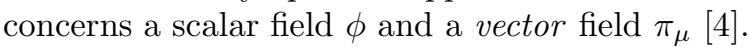


which generates external time translations in the $n$ direction. In coordinates adapted to $n$ these transformations take the form $\phi_{n}(t, \underline{x}) \mapsto \phi_{n}(t+\lambda, \underline{x})$, and similarly for $\pi_{n}(t, \underline{x})$.

There is another notion of time translation in the history theory. Intuitively the Hamiltonian at each instant of external time generates dynamical evolution internal to each fibre of $\xi_{n}$. More precisely, the time-averaged Hamiltonian

$$
H_{n}=\frac{1}{2} \int d^{4} X\left[\pi_{n}^{2}+\left(\eta^{\mu \nu}-n^{\mu} n^{\nu}\right) \partial_{\mu} \phi_{n} \partial_{\nu} \phi_{n}+m^{2} \phi_{n}^{2}\right]
$$

generates transformations $\phi_{n}(X) \mapsto \phi_{n}(X, s)$.

The action operator is made up of the Louville and Hamiltonian operators as follows:

$$
S_{n}:=V_{n}-H_{n}
$$

and the equations of motion can be written in the form

$$
\begin{aligned}
& \left\{S_{n}, \phi_{n}(X)\right\}=0 \\
& \left\{S_{n}, \pi_{n}(X)\right\}=0
\end{aligned}
$$

\subsubsection{Poincare covariance}

Savvidou [7] has shown the existence of two Poincare groups in the histories formulation of the scalar field. The $n$-spatial components of the two groups are identical, but the time translations of the internal Poincare group are generated by the Hamiltonian while the time translations of the external Poincare group are generated by the Louville operator.

External boosts correspond to the following automorphism of the history algebra:

$$
\begin{aligned}
& \phi_{n}(t, \underline{x}, 0) \mapsto \phi_{\Lambda n}(\Lambda(t, \underline{x}), 0) \\
& \pi_{n}(t, \underline{x}, 0) \mapsto \pi_{\Lambda n}(\Lambda(t, \underline{x}), 0)
\end{aligned}
$$

where we have used adapted coordinates to write $\phi_{n}(X, 0)=\phi_{n}(t, \underline{x}, 0)$ and $\Lambda(t, \underline{x})$ denotes the usual Lorentz transformations acting on inertial coordinates $(t, \underline{x})$. We note that, in the classical case, these automorphisms cannot be generated by canonical transformations. This is because there is no momentum conjugate to the foliation vector $n$, and thus no way to generate 
changes in $n$. The 'multisymplectic' approach [4] offers a solution to this problem in the classical theory, and in the quantum theory changes in $n$ correspond to mapping between inequivalent representations of the history algebra.

Internal boosts act on the fields as follows:

$$
\begin{aligned}
& \phi_{n}(0, \underline{x}, s) \mapsto \phi_{n}(0, \Lambda(\underline{x}, s)) \\
& \pi_{n}(0, \underline{x}, s) \mapsto \pi_{n}(0, \Lambda(\underline{x}, s))
\end{aligned}
$$

As the internal boosts leave the foliation vector fixed they can be implemented by canonical transformations. The generator of internal boosts on the hyperplane $s=$ const is

$$
{ }^{i n t} K_{n}(m)=m_{\mu} \int d^{4} X\left[\pi s \partial^{\mu} \phi-X^{\mu} H_{n}(X)\right]
$$

where $H_{n}(X)$ is the Hamiltonian density and the integral is over the surface $s=$ const.

The fields $\phi_{n}(X, s)$ are defined on an extended space-time $\mathcal{N}=\mathcal{M} \times \mathbb{R}$. However, the theory is not invariant under the full $S O(2,3)$ isometry group of this space-time. This is evident from the fact that the algebra (舟), (5), (6) is defined on external space-time, that is the surface in $\mathcal{N}$ defined by $s=0$, and not on internal space-time (defined by $n \cdot X=0$ ). Also, the generators of the symmetries are defined as integrals over external space-time. This indicates that the fields are not true scalar fields on $\mathcal{N}$. However, the fields are invariant under the internal and external $S O(1,3)$ subgroups of $S O(2,3)$.

In the case of the scalar field these subtleties can be overlooked, but the construction of a history theory of vector fields acutely illustrates this issue. A particularly relevant question is whether the history vector field should have 4 indices, or 5 as it must to be a vector field on $\mathcal{N}$.

\section{Massive vector field}

\subsection{State space theory}

In this section we give a brief overview of the standard state space theory of the massive vector field on $\mathcal{M}$. 
We begin with the covariant theory. The covariant configuration space is $\mathcal{X}(\mathcal{M})$, the space of vector fields on $\mathcal{M}$. The massive vector field is described by the following Lagrangian [11]:

$$
\mathcal{L}=-\frac{1}{4} \phi_{\mu \nu} \phi^{\mu \nu}+\frac{1}{2} m^{2} \phi_{\mu} \phi^{\mu}
$$

where $\phi^{\mu \nu}(X):=\partial^{[\mu} \phi^{\nu]}(X)$ and $\phi \in \mathcal{X}(\mathcal{M})$. The resulting field equations are

$$
\begin{aligned}
\left(\square+m^{2}\right) \phi^{\mu}(X) & =0 \\
\partial_{\mu} \phi^{\mu}(X) & =0 .
\end{aligned}
$$

The first of these equations shows that each component of the field behaves like a massive scalar field. The second equation is known as the Fierz-Pauli equation and it is the first indication of the presence of constraints in the theory.

To pass to the canonical theory we choose a Cauchy surface in $\mathcal{M}$ and consider the fields on this Cauchy surface. More precisely, we choose a spacelike embedding $\iota: \Sigma \rightarrow \mathcal{M}$, where $\Sigma \simeq \mathbb{R}^{3}$, and take the corresponding configuration space to be the space of fields $\phi_{\iota}^{\mu}(\underline{x})$ where $\underline{x} \in \iota(\Sigma)$. However the fields $\phi_{\iota}^{\mu}(\underline{x})$ are not geometric objects on either $\Sigma$ or $\mathcal{M}$. The geometrical interpretation of the fields is clarified by considering $\operatorname{Emb}(\Sigma, \mathcal{M})$, the space of embeddings of $\Sigma$ into $\mathcal{M}$. Then $\phi_{\iota}$ can be thought of as an element of $T_{\iota} \operatorname{Emb}(\Sigma, \mathcal{M})$ where the tangent space to $\operatorname{Emb}(\Sigma, \mathcal{M})$ at the embedding $\iota$ is defined as

$$
T_{\iota} \operatorname{Emb}(\Sigma, \mathcal{M})=\left\{\psi: \Sigma \rightarrow T \mathcal{M} \mid \psi(\underline{x}) \in T_{\iota(\underline{x})} \mathcal{M}\right\}
$$

The configuration space of the canonical theory is then defined as $Q_{\iota}:=$ $T_{\iota} \operatorname{Emb}(\Sigma, \mathcal{M})$ for some fixed $\iota$. The cotangent space of $\operatorname{Emb}(\Sigma, \mathcal{M})$ at $\iota$ is defined similarly:

$$
T_{\iota}^{*} \operatorname{Emb}(\Sigma, \mathcal{M})=\left\{l: \Sigma \rightarrow T^{*} \mathcal{M} \mid l(\underline{x}) \in T_{\iota(\underline{x})}^{*} \mathcal{M}\right\}
$$

and the pairing between these two spaces is given by

$$
<l, \psi>_{\iota}=\int_{\Sigma} d \theta_{\underline{x}} l_{\mu}(\iota(\underline{x})) \psi^{\mu}(\iota(\underline{x}))
$$


where $d \theta_{\underline{x}}$ is an arbitrary volume element on $\Sigma$. The state space $P_{\iota}$ is the cotangent bundle of $Q_{\iota}$, and can be identified with the Cartesian product $Q_{\iota} \times T_{\iota}^{*} \operatorname{Emb}(\Sigma, \mathcal{M})$.

As we are considering flat space-time, there exists a family of preferred embeddings; those which correspond to inertial frames. The space of preferred embeddings can be parametrised by pairs $(n, t)$ where $n$ is a future pointing unit vector in $\mathcal{M}$ and $t \in \mathbb{R}$. As we are considering a deterministic system, we choose $t=0$ without loss. We denote the configuration space and the state space corresponding to the embedding labeled by $(n, 0)$ as $Q_{n}$ and $P_{n}$ respectively. The state space $P_{n}$ carries the following Poisson algebra;

$$
\begin{aligned}
& \left\{\phi_{n}^{\mu}(\underline{x}), \phi_{n}^{\nu}\left(\underline{x}^{\prime}\right)\right\}=0 \\
& \left\{\pi_{\mu}^{n}(\underline{x}), \pi_{\nu}^{n}\left(\underline{x}^{\prime}\right)\right\}=0 \\
& \left\{\phi_{n}^{\mu}(\underline{x}), \pi_{\nu}^{n}\left(\underline{x}^{\prime}\right)\right\}=\delta_{\nu}^{\mu} \delta_{n}^{(3)}\left(\underline{x}-\underline{x}^{\prime}\right)
\end{aligned}
$$

where $\delta_{n}^{(3)}\left(\underline{x}-\underline{x}^{\prime}\right)$ is the delta function on $\Sigma_{n}$.

A field $\phi_{n}^{\mu}(\underline{x}) \in Q_{n}$ can be decomposed into the pair $\left(\phi_{n}^{t}(\underline{x}),{ }^{n} \phi^{\mu}(\underline{x})\right)$ where

$$
\begin{aligned}
\phi_{n}^{t}(\underline{x}) & :=n_{\mu} \phi_{n}^{\mu}(\underline{x}) \\
{ }^{n} \phi^{\mu}(\underline{x}) & :={ }^{n} P_{\nu}^{\mu} \phi_{n}^{\nu}(\underline{x})
\end{aligned}
$$

and we have introduced the $n$-spatial projection tensor defined by

$$
{ }^{n} P_{\nu}^{\mu}:=\delta_{\nu}^{\mu}-n^{\mu} n_{\nu}
$$

It follows that $n_{\mu}{ }^{n} P_{\nu}^{\mu}=0$ and $n^{\nu}{ }^{n} P_{\nu}^{\mu}=0$. The fields $\phi_{n}^{t}(\underline{x})$ and ${ }^{n} \phi^{\mu}(\underline{x})$ are defined on the space of embeddings, but $\phi_{n}^{t}(\underline{x})$ pulls back to give a scalar field on $\Sigma_{n}$. We can use the metric on $\mathcal{M}$ to lower the index on ${ }^{n} \phi^{\mu}(\underline{x})$. The resulting one-form can be pulled back to $\Sigma_{n}$, and then the index can be raised using the metric on $\Sigma_{n}$ to give a vector field on $\Sigma_{n}$. In a similar way, objects defined by ${ }^{n} \pi_{\mu}(\underline{x}):={ }^{n} P_{\mu}^{\nu} \pi_{\nu}^{n}(\underline{x})$, and ${ }^{n} \phi^{\mu \nu}(\underline{x}):={ }^{n} P_{\sigma}^{\mu n} P_{\rho}^{\nu} \phi_{n}^{\sigma \rho}(\underline{x})$ can be thought of as a one-form and a covariant tensor on $\Sigma_{n}$.

The canonical momenta are computed from the Lagrangian and turn out to be [12

$$
\pi_{t}^{n}(\underline{x})=0,{ }^{n} \pi_{\mu}(\underline{x})={ }^{n} P_{\mu}^{\rho} n^{\nu} \phi_{\nu \rho}(\underline{x})
$$

\footnotetext{
${ }^{4}$ Although this is an inherently non-linear process, it presents no extra difficulties in the case of Minkowski space-time.
} 
The first of these equations is a primary constraint. The canonical Hamiltonian is computed to be

$$
\begin{array}{r}
H_{n}=\int_{\Sigma_{n}} d \theta_{\underline{x}}\left[\frac{1}{2}{ }^{n} \pi_{\mu}{ }^{n} \pi^{\mu}-\frac{1}{4}{ }^{n} \phi_{\mu \nu}{ }^{n} \phi^{\mu \nu}-\frac{1}{2} m^{2}\left(\phi_{n}^{t}\right)^{2}\right. \\
\left.+\frac{1}{2} m^{2 n} \phi_{\mu}{ }^{n} \phi^{\mu}-\phi_{n}^{t}{ }^{n} \partial^{\mu n} \pi_{\mu}\right]
\end{array}
$$

where the $n$-spatial derivative is defined as ${ }^{n} \partial^{\mu}={ }^{n} P_{\nu}^{\mu} \partial^{\nu}$. For the primary constraint $\pi_{t}^{n}(\underline{x})=0$ to be preserved by the dynamical evolution, it is necessary and sufficient that $\left\{H_{n}, \pi_{t}^{n}(\underline{x})\right\}=0$. This implies the secondary constraint

$$
m^{2} \phi_{n}^{t}(\underline{x})+{ }^{n} \partial^{\mu n} \pi_{\mu}(\underline{x})=0 .
$$

A point in the constraint surface determines and is fully determined by the pair $\left({ }^{n} \pi_{\mu}(\underline{x}),{ }^{n} \phi^{\nu}(\underline{x})\right)$. The constraint surface is therefore isomorphic to the space $C_{n}=\Lambda^{1}\left(\Sigma_{n}\right) \times \mathcal{X}\left(\Sigma_{n}\right)$ where $\Lambda^{1}\left(\Sigma_{n}\right)$ is the space of 1-forms on $\Sigma_{n}$.

The constraints do not commute under the Poisson bracket and so they form a second class pair. This implies that the pull-back of the symplectic 2 -form on $P_{n}$ to the constraint surface is non-degenerate. Therefore there is a well-defined Poisson algebra on the constraint surface which is given by the Dirac brackets:

$$
\left\{{ }^{n} \phi^{\mu}(\underline{x}),{ }^{n} \pi_{\nu}\left(\underline{x}^{\prime}\right)\right\}_{D}={ }^{n} P_{\nu}^{\mu} \delta^{(3)}\left(\underline{x}-\underline{x}^{\prime}\right)
$$

\subsection{Classical History theory}

We begin with the abstract state space $P$, and follow the usual procedure of taking a one-parameter family of copies of $P$. This results in a trivial vector bundle $\xi_{n}: P \times \mathbb{R} \rightarrow \mathbb{R}$ where the fibre $\xi_{n}^{-1}(t)=P_{n, t}$ for each $t$. Sections of $\xi_{n}$ correspond to histories of the vector field with respect to the foliation labelled by $n$. Thus a history is a map

$$
h_{n}: t \mapsto\left(\phi_{n}^{\mu}(t ; \underline{x}), \pi_{\nu}^{n}(t ; \underline{x})\right)
$$

If we choose a volume element on $\mathbb{R}$ then a symplectic structure is induced on the space of maps $\mathbb{R} \rightarrow P$ because $P$ is a symplectic manifold. The symplectic structure defines the following algebra

$$
\begin{aligned}
& \left\{\phi_{n}^{\mu}(t ; \underline{x}), \phi_{n}^{\nu}\left(t^{\prime} ; \underline{x}^{\prime}\right)\right\}=0 \\
& \left\{\pi_{\mu}^{n}(t ; \underline{x}), \pi_{\nu}^{n}\left(t^{\prime} ; \underline{x}^{\prime}\right)\right\}=0 \\
& \left\{\phi_{n}^{\mu}(t ; \underline{x}), \pi_{\nu}^{n}\left(t^{\prime} ; \underline{x}^{\prime}\right)\right\}=\delta_{\nu}^{\mu} \delta\left(t-t^{\prime}\right) \delta_{n}^{(3)}\left(\underline{x}-\underline{x}^{\prime}\right)
\end{aligned}
$$


In the case of the scalar field a one-parameter family of functions on $\Sigma_{n}$ :

$$
t \mapsto \phi_{n}(t ; \underline{x})
$$

was identified with a function $\phi_{n}(X)$ on $\mathcal{M}$. In a similar way, for a fixed foliation, a one-parameter family of elements of $Q_{n}$

$$
t \mapsto \phi_{n}^{\mu}(t ; \underline{x})
$$

is equivalent to a unique vector field $\phi_{n}^{\mu}(X) \in \mathcal{X}(\mathcal{M})$. The one-parameter family $t \mapsto \pi_{\mu}^{n}(t ; \underline{x})$ can be identified with a one-form $\pi_{\mu}^{n}(X) \in \Lambda^{1}(\mathcal{M})$ in the same way, and these fields satisfy the covariant looking algebra

$$
\begin{aligned}
& \left\{\phi_{n}^{\mu}(X), \phi_{n}^{\nu}\left(X^{\prime}\right)\right\}=0 \\
& \left\{\pi_{\mu}^{n}(X), \pi_{\nu}^{n}\left(X^{\prime}\right)\right\}=0 \\
& \left\{\phi_{n}^{\mu}(X), \pi_{\nu}^{n}\left(X^{\prime}\right)\right\}=\delta_{\nu}^{\mu} \delta^{(4)}\left(X-X^{\prime}\right)
\end{aligned}
$$

In this way, the history space, $\Pi_{n}$, can be identified with the space $\mathcal{X}(\mathcal{M}) \times$ $\Lambda^{1}(\mathcal{M})$. We can decompose $\phi_{n}^{\mu}(X)$ into the pair $\left(\phi_{n}^{t}(X),{ }^{n} \phi^{\mu}(X)\right)$ defined by

$$
\begin{aligned}
\phi_{n}^{t}(X) & :=n_{\mu} \phi_{n}^{\mu}(X) \\
{ }^{n} \phi^{\mu}(X) & :={ }^{n} P_{\nu}^{\mu} \phi_{n}^{\nu}(X)
\end{aligned}
$$

and we use these fields to define the generators of internal and external time translations. External time translations are generated by the 'Louville' operator,

$$
V_{n}:=\int d^{4} X\left[\pi_{t}^{n} n_{\nu} \partial^{\nu} \phi_{n}^{t}+{ }^{n} \pi_{\mu} n_{\nu} \partial^{\nu n} \phi^{\mu}\right]
$$

Internal time translations are generated by the time-averaged Hamiltonian

$$
\begin{array}{r}
H_{n}=\int d^{4} X\left[\frac{1}{2} \pi_{\mu}^{n} \pi^{\mu}+\frac{1}{4}{ }^{n} \phi_{\mu \nu}{ }^{n} \phi^{\mu \nu}-\frac{1}{2} m^{2}\left(\phi_{n}^{t}\right)^{2}\right. \\
\left.+\frac{1}{2} m^{2 n} \phi_{\mu}{ }^{n} \phi^{\mu}-\phi_{n}^{t}{ }^{n} \partial^{\mu n} \pi_{\mu}\right]
\end{array}
$$

The internal time translations generated by $H_{n}$ take the form

$$
\phi_{n}^{\mu}(X) \mapsto \phi_{n}^{\mu}(X, s)
$$

In the next subsection we will consider the geometric meaning of these curious objects which have four components, but depend on five space-time variables. 
In the remainder of this subsection we discuss the history constraint surface $\mathcal{C}_{n} \subset \Pi_{n}$. An arbitrary element of $\Pi_{n}$ will not be compatible with the constraints. The constraint submanifold, $\mathcal{C}_{n}$, contains all elements of $\Pi$ which satisfy the constraints:

$$
\pi_{t}^{n}(X)=0, m^{2} \phi_{n}^{t}(X)+{ }^{n} \partial^{\mu n} \pi_{\mu}(X)=0
$$

Thus a point in $\mathcal{C}_{n}$ is equivalent to a pair $\left({ }^{n} \phi^{\mu}(X),{ }^{n} \pi_{\nu}(X)\right)$. The Poisson algebra induced on $\mathcal{C}_{n}$ by pulling back along the natural inclusion map $\mathcal{C}_{n} \hookrightarrow$ $\Pi_{n}$ is given by

$$
\begin{aligned}
& \left\{{ }^{n} \phi^{\mu}(X),{ }^{n} \phi^{\nu}\left(X^{\prime}\right)\right\}=0 \\
& \left\{{ }^{n} \pi_{\mu}(X),{ }^{n} \pi_{\nu}\left(X^{\prime}\right)\right\}=0 \\
& \left\{{ }^{n} \phi^{\mu}(X),{ }^{n} \pi_{\nu}\left(X^{\prime}\right)\right\}={ }^{n} P_{\nu}^{\mu} \delta^{(4)}\left(X-X^{\prime}\right)
\end{aligned}
$$

and we note that $\mathcal{C}_{n}$ is diffeomorphic to the space of sections of the bundle $C_{n} \times \mathbb{R} \rightarrow \mathbb{R}$.

\subsection{Poincare covariance}

As discussed in the previous section for the case of the scalar field, the notion of two times leads naturally to the definition of two Poincare groups. The external Poincare group mixes the external time with the $n$-spatial variables $\underline{x}$ and the internal Poincare group mixes internal time with $\underline{x}$. The crucial new feature of the generators for the vector field is the mixing of the time-like and space-like components of the field.

\subsubsection{External Poincare group}

The generators of external space-time translations can be written in covariant looking form as

$$
{ }^{e x t} P_{n}^{\mu}=\int d^{4} X \pi_{\nu}^{n}(X) \partial^{\mu} \phi_{n}^{\nu}(X)
$$

Next we define

$$
M_{n}^{\mu \nu}=\int d^{4} X \pi_{\rho}^{n}(X)\left(X^{\mu} \partial^{\nu}-X^{\nu} \partial^{\mu}\right) \phi_{n}^{\rho}(X)+\sigma_{n}^{\mu \nu}
$$

where the 'spin tensor' is

$$
\sigma_{n}^{\mu \nu}=\int d^{4} X \pi_{\rho}^{n}(X)\left(\delta^{\rho \mu} \delta_{\sigma}^{\nu}-\delta^{\rho \nu} \delta_{\sigma}^{\mu}\right) \phi_{n}^{\sigma}(X)
$$


The generators of $n$-spatial rotations can be parametrised by two vectors $m^{1}$ and $m^{2}$ satisfying $m^{1} \cdot n=m^{2} \cdot n=0$ in the following way:

$$
M_{n}\left(m^{1}, m^{2}\right)=m_{\mu}^{1} m_{\nu}^{2} M_{n}^{\mu \nu}
$$

As in the case of the scalar field, the external boosts cannot be implemented by canonical transformations because of the change in foliation. The natural definition of the automorphisms generated by the action of the external boosts is

$$
\begin{aligned}
\phi_{n}^{\mu}(t, \underline{x}, 0) & \mapsto \Lambda_{\nu}^{\mu} \phi_{\Lambda n}^{\nu}(\Lambda(t, \underline{x}), 0) \\
\pi_{\mu}^{n}(t, \underline{x}, 0) & \mapsto \Lambda_{\mu}^{\nu} \pi_{\nu}^{\Lambda n}(\Lambda(t, \underline{x}), 0)
\end{aligned}
$$

So the external boosts mix $t$ with $\underline{x}$, and the external time component $\phi_{\Lambda n}^{t}(t, \underline{x}, 0)$ with the space-like components ${ }^{\Lambda n} \phi^{\mu}(t, \underline{x}, 0)$.

\subsubsection{Internal Poincare group}

The rotation and spatial translation generators of the internal Poincare group coincide with those for the external Poincare group. However, internal time translations are generated by the Hamiltonian, and therefore act internally as $\phi_{n}^{\mu}(X) \mapsto \phi_{n}^{\mu}(X, s)$. We tentatively define the generator of internal Lorentz transformations on the $s=$ const hyperplane in the 'obvious' way:

$$
{ }^{i n t} K_{n}(m):=m_{\mu} \int d^{4} X\left[\pi_{\nu}^{n} s \partial^{\mu} \phi_{n}^{\nu}-X^{\mu} H_{n}(X)\right]+n_{\mu} m_{\nu} \sigma^{\mu \nu}
$$

where $H_{n}(X)$ is the Hamiltonian density, $m$ is a vector satisfying $m \cdot n=0$, and the integral is over the surface $s=$ const. This functional generates the automorphisms

$$
\begin{aligned}
\phi_{n}^{\mu}(0, \underline{x}, s) & \mapsto \Lambda_{\nu}^{\mu} \phi_{n}^{\nu}(0, \Lambda(\underline{x}, s)) \\
\pi_{\mu}^{n}(0, \underline{x}, s) & \mapsto \Lambda_{\mu}^{\nu} \pi_{\nu}^{n}(0, \Lambda(\underline{x}, s))
\end{aligned}
$$

${ }^{i n t} K_{n}(m)$ mixes $s$ with $\underline{x}$, and $\phi_{n}^{t}(0, \underline{x}, s)$ with ${ }^{n} \phi_{n}^{\mu}(0, \underline{x}, s)$. So in this transformation, the function $\phi_{n}^{t}(0, \underline{x}, s)$ is associated with the internal time direction, whereas it was associated with the external time direction by the external boosts.

\footnotetext{
${ }^{5} i$.e., rotations that leave $n$ fixed if $n$ is considered as a space-time vector rather than as the foliation label.
} 


\subsection{Alternative interpretations of the history vector field}

The above discussion suggests that it is misleading to think of the history vector fields as a family of 4 -vectors on external space-time. We propose two alternative interpretations of the history vector fields.

\subsubsection{Four-component fields}

One way of thinking of the history fields is as a family of 4 -vectors, but with the temporal component in the $\partial_{\tau}:=\partial_{s}+\partial_{t}$ direction;

$$
\phi_{n}=\phi_{n}^{\tau}(X, s) \partial_{\tau}+\phi_{n}^{i}(X, s) \partial_{i}
$$

where $\phi_{n}^{\tau}(X, s)=\phi_{n}^{t}(X, s)$ and we have used coordinates adapted to $n$. From this perspective it is natural to look for a representation of the Poincare group in which the boosts act in this direction. The orbital part of the internal boost generator on $s=0$ would be

$$
K_{n}(m):=\int d^{4} X\left[n \cdot X \pi_{\mu}^{n}(X) m_{\nu} \partial^{\nu} \phi_{n}^{\mu}(X)-m \cdot X\left(V_{n}(X)+H_{n}(X)\right)\right]
$$

where $V_{n}(X)$ is the 'Louville' density. However, it can be shown that

$$
\left\{K_{n}\left(m^{1}\right), K_{n}\left(m^{2}\right)\right\} \neq m_{\mu}^{1} m_{\nu}^{2} M_{n}^{\mu \nu}
$$

even on the solutions to the equations of motion, and so the functionals $K_{n}(m)$ do not form a representation of the Poincare group.

Thus it is not possible to eliminate the two times in favour of one 'physical' time direction $\partial_{\tau}$, in a covariant way. Nevertheless, $\tau$ does have a special significance in the theory. This is indicated by the equations of motion. Returning to the example of the scalar field for a moment; if $\phi_{n}(X)$ is a solution then $\left\{S_{n}, \phi_{n}(X)\right\}=0$ implies that

$$
\left.\left(\partial_{s}-\partial_{t}\right) \phi_{n}(X)\right|_{s=0}=0
$$

and so all the temporal change in such histories occurs in the $\tau$ direction. 


\subsubsection{Five-component fields}

We can augment $\phi_{n}^{\mu}(X, s)$ with a new degree of freedom $\phi_{n}^{s}(X, s)$ to form $\tilde{\phi}_{n}^{M}(X, s)$, a five component 'vector field' on the extended space-time $\mathcal{N}=$ $\mathcal{M} \times \mathbb{R}$ with metric $\operatorname{diag}(+,-,-,-,+)$. The extended fields are written

$$
\tilde{\phi}_{n}=\tilde{\phi}_{n}^{M}(X, s) \partial_{M}, \tilde{\pi}^{n}=\tilde{\pi}_{M}^{n}(X, s) d x^{M}
$$

The label $M$ runs over $t, 1,2,3, s$, where we define $M=t$ to refer to external time, $M=1,2,3$ to correspond to the spatial directions, and $M=s$ to refer to internal time.

The extended history space, $\tilde{\Pi}_{n} \subset \mathcal{X}(\mathcal{N}) \times \Lambda^{1}(\mathcal{N})$, contains all pairs $\left(\tilde{\phi}_{n}, \tilde{\pi}_{n}\right) \in \mathcal{X}(\mathcal{N}) \times \Lambda^{1}(\mathcal{N})$ that satisfy the internal field equations:

$$
\begin{aligned}
\partial_{s} \tilde{\phi}_{n}^{M}(X, s) & =\left\{\tilde{H}_{n}, \tilde{\phi}_{n}^{M}(X, s)\right\} \\
\partial_{s} \tilde{\pi}_{M}^{n}(X, s) & =\left\{\tilde{H}_{n}, \tilde{\pi}_{M}^{n}(X, s)\right\}
\end{aligned}
$$

where $\tilde{H}_{n}$ is the Hamiltonian on extended history space, and is defined in the next section.

The history algebra can be extended to these fields in a natural way:

$$
\left\{\tilde{\phi}_{n}^{M}(X), \tilde{\pi}_{N}^{n}\left(X^{\prime}\right)\right\}=\delta_{N}^{M} \delta^{(4)}\left(X-X^{\prime}\right)
$$

This defines the algebra of the fields on the surface $s=0$, which is a submanifold of $\mathcal{N}$. Hamiltonian evolution can be used to extend this definition to the rest of $\mathcal{N}$. The fact that the algebra is naturally defined on the hyperplane $s=0$ and not on the hyperplane $n \cdot X=0$ reflects the underlying asymmetry between the two 'modes' of time. As a consequence of this asymmetry, the theory constructed from the fields $\tilde{\phi}_{n}$ will not be covariant under the full $S O(2,3)$ isometry group associated with $\mathcal{N}$. In particular it will not be covariant under the action of the $S O(2)$ subgroup acting in the $(s, t)$ plane.

In order to discuss the Poincare transformations of the five-component fields, we make the definition:

$$
\tilde{M}_{n}^{M N}=\int_{\mathcal{M}^{(e)}} d^{4} X \tilde{\pi}_{R}^{n}(X)\left(X^{M} \partial^{N}-X^{N} \partial^{M}\right) \tilde{\phi}_{n}^{R}(X)+\tilde{\sigma}_{n}^{M N}
$$

where $\mathcal{M}^{(e)} \subset \mathcal{N}$ is external space-time, defined as the surface $s=0$, and the extended spin tensor is defined as

$$
\tilde{\sigma}_{n}^{M N}=\int_{\mathcal{M}^{(e)}} d^{4} X \tilde{\pi}_{A}^{n}(X)\left(\delta^{A M} \delta_{B}^{N}-\delta^{A N} \delta_{B}^{M}\right) \tilde{\phi}_{n}^{B}(X)
$$


Using $\tilde{M}_{n}^{M N}$ we can write the rotation generators as

$$
\tilde{M}_{n}\left(\tilde{m}^{1}, \tilde{m}^{2}\right)=\tilde{m}_{M}^{1} \tilde{m}_{N}^{2} \tilde{M}_{n}^{M N}
$$

where $\tilde{m}^{1}$ and $\tilde{m}^{2}$ are $n$-spatial in the sense that $\tilde{m}_{M}^{1} \tilde{n}^{M}=\tilde{m}_{M}^{1} \tilde{e}^{M}=0$, and similarly for $\tilde{m}^{2}$.

The foliation vector $n$ is an element of the external space-time which is a subspace of $\mathcal{N}$. Using the canonical embedding, $n$ can be considered as a vector in $\mathcal{N}$, which we denote by $\tilde{n}$ and is given in coordinates $(X, s)$ by $(n, 0)$. Using this coordinate system the internal future pointing unit vector $\tilde{e}$ can be written as $(0,1)$. We can use these vectors to decompose an extended field $\tilde{\phi}_{n}^{M}(X, s)$ into its external and internal time components as follows:

$$
\tilde{\phi}_{n}^{t}(X, s)=\tilde{n}_{M} \tilde{\phi}_{n}^{M}(X, s), \tilde{\phi}_{n}^{s}(X, s)=\tilde{e}_{M} \tilde{\phi}_{n}^{M}(X, s)
$$

Finally, given three orthogonal, $n$-spatial, unit vectors in $\mathcal{N}, \tilde{m}^{i}$, where $i=$ $1,2,3$, the spatial components of $\tilde{\phi}_{n}^{M}(X, s)$ are

$$
\tilde{\phi}_{n}^{i}(X, s)=\tilde{m}_{M}^{i} \tilde{\phi}_{n}^{M}(X, s)
$$

Using this basis we write the action of the external boosts as

$$
\begin{aligned}
\tilde{\phi}_{n}^{s}(t, \underline{x}, 0) & \mapsto \tilde{\phi}_{\Lambda n}^{s}(\Lambda(t, \underline{x}), 0) \\
\tilde{\phi}_{n}^{\mu}(t, \underline{x}, 0) & \mapsto \Lambda_{\nu}^{\mu} \tilde{\phi}_{\Lambda n}^{\nu}(\Lambda(t, \underline{x}), 0) \\
\tilde{\pi}_{s}^{n}(t, \underline{x}, 0) & \mapsto \tilde{\pi}_{s}^{\Lambda n}(\Lambda(t, \underline{x}), 0) \\
\tilde{\pi}_{\mu}^{n}(t, \underline{x}, 0) & \mapsto \Lambda_{\mu}^{\nu} \tilde{\pi}_{\nu}^{\Lambda n}(\Lambda(t, \underline{x}), 0)
\end{aligned}
$$

where $\mu$ takes the values $t, 1,2,3$.

Internal boosts are generated by

$$
{ }^{i n t} \tilde{K}_{n}(\tilde{m}):=\tilde{m}_{M} \int_{\mathcal{M}_{s}^{(e)}} d^{4} X\left[\tilde{\pi}_{N}^{n} s \partial^{M} \tilde{\phi}_{n}^{N}-X^{M} \tilde{H}_{n}(X)\right]+\tilde{e}_{M} \tilde{m}_{N} \tilde{\sigma}_{n}^{M N}
$$

and the resulting automorphisms are

$$
\begin{aligned}
\tilde{\phi}_{n}^{t}(0, \underline{x}, s) & \mapsto \tilde{\phi}_{n}^{t}(0, \Lambda(\underline{x}, s)) \\
\tilde{\phi}_{n}^{\bar{\mu}}(0, \underline{x}, s) & \mapsto \Lambda_{\bar{\nu}}^{\bar{\phi}} \tilde{\phi}_{n}^{\bar{\nu}}(0, \Lambda(\underline{x}, s)) \\
\tilde{\pi}_{t}^{n}(0, \underline{x}, s) & \mapsto \tilde{\pi}_{t}^{n}(0, \Lambda(\underline{x}, s)) \\
\tilde{\pi}_{\bar{\mu}}^{n}(0, \underline{x}, s) & \mapsto \Lambda_{\bar{\mu}}^{\bar{\nu}} \tilde{\pi}_{\bar{\nu}}^{n}(0, \Lambda(\underline{x}, s))
\end{aligned}
$$


where $\bar{\mu}$ takes the values $1,2,3, s$. Now the components of $\tilde{\phi}_{n}$ and $\tilde{\pi}^{n}$ are mixed in a way which is consistent with the mixing of the space-time variables. This indicates that the extended fields are an appropriate way of thinking about the history fields. However, it should be emphasised that the extended fields are not covariant under $S O(2,3)$. Let $V^{M}$ denote the vector defined by the field $\tilde{\phi}_{n}^{M}(X, s)$ at the point $(X, s)$. Using the basis $\left(\tilde{n}, \tilde{e}, \tilde{m}^{i}\right)$, $V^{M}$ can be decomposed into an 'external' four-vector $\left(V^{t}, V^{i}\right)$, or into an 'internal' four-vector $\left(V^{s}, V^{i}\right)$, and each of these four-vectors is a covariant object under the appropriate Poincare group. This suggests a third interpretation of the history vector fields as pairs of four-component fields with identical $n$-spatial components. However, this identification is not preserved under the action of the external boosts, so it seems that we are left with the five-vector interpretation as the only viable one.

It remains to be shown that the extra degrees of freedom can be included in the action in a way that is consistent with the symmetries and equations of motion of the theory.

\subsection{The action}

In the case of scalar field theory the physical action functional is written as $S_{n}=V_{n}-H_{n}$. The Louville operator is associated with external time in the sense that it generates translations in the external time direction. In the same way, the Hamiltonian is associated with internal time, and the action functional mixes the two 'modes' of time.

First we will need the following definition: The $n$-spatial part of $\tilde{\phi}_{n}^{M}(X, s)$ is defined as

$$
{ }^{n} \tilde{\phi}^{M}(X, s)={ }^{n} \tilde{P}_{N}^{M} \tilde{\phi}^{N}(X, s)
$$

where the extended $n$-spatial projection tensor is

$$
{ }^{n} \tilde{P}_{N}^{M}:=\delta_{N}^{M}-\tilde{n}^{M} \tilde{n}_{N}-\tilde{e}^{M} \tilde{e}_{N}
$$

Similarly we define ${ }^{n} \partial^{M}:={ }^{n} \tilde{P}_{N}^{M} \partial^{N}$.

Let $\tilde{V}_{n}$ denote the extension of the Louville operator to the extended fields. $\tilde{V}_{n}$ is defined in the following natural way,

$$
\tilde{V}_{n}:=\int_{\mathcal{M}^{(e)}} d^{4} X\left[\tilde{\pi}_{s}^{n} \partial_{n}^{t} \tilde{\phi}_{n}^{s}+\tilde{\pi}_{t}^{n} \partial_{n}^{t} \tilde{\phi}_{n}^{t}+{ }^{n} \tilde{\pi}_{M} \partial_{n}^{t n} \tilde{\phi}^{M}\right]
$$


where $\partial_{n}^{t}=\tilde{n}_{M} \partial^{M}$ is the derivative in the external time direction defined by $\tilde{n}$. The extended Hamiltonian, $\tilde{H}_{n}$, is defined as

$$
\begin{aligned}
\tilde{H}_{n}:=\int_{\mathcal{M}^{(e)}} & d^{4} X\left[\frac{1}{2} n \tilde{\pi}_{M}{ }^{n} \tilde{\pi}^{M}+\frac{1}{4}{ }^{n} \tilde{\phi}_{M N}{ }^{n} \tilde{\phi}^{M N}-\frac{1}{2} m^{2}\left(\tilde{\phi}_{n}^{s}\right)^{2}\right. \\
& \left.-\frac{1}{2} m^{2}\left(\tilde{\phi}_{n}^{t}\right)^{2}+\frac{1}{2} m^{2}{ }^{n} \tilde{\phi}_{M}{ }^{n} \tilde{\phi}^{M}-\tilde{\phi}_{n}^{t}{ }^{n} \partial^{M}{ }^{n} \tilde{\pi}_{M}\right]
\end{aligned}
$$

The important thing about this Hamiltonian is that $\tilde{\phi}_{n}^{s}$ and $\tilde{\phi}_{n}^{t}$ both appear in the mass term, but only $\tilde{\phi}_{n}^{t}$ appears as the coefficient of ${ }^{n} \partial^{M}{ }^{n} \tilde{\pi}_{M}$. Due to this asymmetry between $\tilde{\phi}_{n}^{s}$ and $\tilde{\phi}_{n}^{t}$, the Hamiltonian is not invariant under $S O(2,3)$. The action is defined to be $\tilde{S}_{n}:=\tilde{V}_{n}-\tilde{H}_{n}$. The resulting field equations are,

$$
\begin{array}{rlll}
\left\{\tilde{S}_{n}, \tilde{\phi}_{n}^{s}\right\} & =0 & \Rightarrow & \partial_{n}^{t} \tilde{\phi}_{n}^{s}=0 \\
\left\{\tilde{S}_{n}, \tilde{\pi}_{s}^{n}\right\}=0 & \Rightarrow & \partial_{n}^{t} \tilde{\pi}_{s}^{n}+m^{2} \tilde{\phi}_{n}^{s}=0 \\
\left\{\tilde{S}_{n}, \tilde{\phi}_{n}^{t}\right\}=0 & \Rightarrow & \partial_{n}^{t} \tilde{\phi}^{t}=0 \\
\left\{\tilde{S}_{n}, \tilde{\pi}_{t}^{n}\right\}=0 & \Rightarrow & \partial_{n}^{t} \tilde{\pi}_{t}^{n}+m^{2} \tilde{\phi}_{n}^{t}+{ }^{n} \partial^{M}{ }^{n} \tilde{\pi}_{M}=0 \\
\left\{\tilde{S}_{n},{ }^{n} \tilde{\phi}^{M}\right\} & =0 & \Rightarrow & \partial_{n}^{t}{ }^{n} \tilde{\phi}^{M}-\left({ }^{n} \tilde{\pi}^{M}+{ }^{n} \partial^{M} \tilde{\phi}_{n}^{t}\right)=0 \\
\left\{\tilde{S}_{n},{ }^{n} \tilde{\pi}_{M}\right\} & =0 & \Rightarrow & \partial_{n}^{t}{ }^{n} \tilde{\pi}_{M}-\left({ }^{n} \partial^{N} \tilde{\phi}_{N M}+m^{2 n} \tilde{\phi}_{M}\right)=0
\end{array}
$$

The physical action has not been derived in the usual way from a Lagrangian, so we do not have the usual identification of primary constraints. The field equations do not determine the time-like components of the $\tilde{\pi}$ field, so we augment the equations of motion with the following equations which are interpreted as the primary constraints of the theory.

$$
\tilde{\pi}_{s}^{n}(X)=0, \tilde{\pi}_{t}^{n}(X)=0
$$

We require these constraints to be conserved in internal time which implies the following secondary constraints

$$
\tilde{\phi}_{n}^{s}(X)=0, m^{2} \tilde{\phi}_{n}^{t}(X)+{ }^{n} \partial^{M}{ }^{n} \tilde{\pi}_{M}(X)=0
$$

so in the history theory of the massive vector field there are two pairs of second class constraints. In the state space theory a single pair of constraints allow the theory to be written in a Lorentz covariant way. In the history theory where there are two $S O(1,3)$ symmetry groups, we have to introduce two pairs of constraints in order to have a covariant theory. 
The three functionals, $\tilde{V}_{n}, \tilde{H}_{n}$ and $\tilde{S}_{n}$ are all invariant under the action of the internal Poincare group. However, the external boosts change the foliation with respect to which $\tilde{V}_{n}$ and $\tilde{H}_{n}$ are defined, giving the transformations $\tilde{V}_{n} \mapsto \tilde{V}_{\Lambda n}$ and $\tilde{H}_{n} \mapsto \tilde{H}_{\Lambda n}$. These transformations imply $\tilde{S}_{n} \mapsto \tilde{S}_{\Lambda n}$, and the history theory is covariant under both Poincare groups if we include the internal foliation dependence.

\section{Electromagnetism}

\subsection{State space theory}

In this section we consider vacuum electromagnetism on $\mathcal{M}$. The covariant configuration space for electromagnetism is $Q=\Lambda^{1}(\mathcal{M})$, and the Lagrangian is

$$
\mathcal{L}:=-\frac{1}{4} F_{\mu \nu} F^{\mu \nu}
$$

where $F_{\mu \nu}(X):=\partial_{[\mu} A_{\nu]}(X)$ for $A \in Q$. The covariant equations of motion which follows from this Lagrangian are

$$
F_{, \nu}^{\mu \nu}(X)=0
$$

Given an embedding $\iota: \Sigma \rightarrow \mathcal{M}$, the canonical configuration space is $Q_{\iota}=T_{\iota}^{*} \operatorname{Emb}(\Sigma, \mathcal{M})$ and the state space, $P_{\iota}$, can be identified with $Q_{\iota} \times$ $T_{\iota} \operatorname{Emb}(\Sigma, \mathcal{M})$. The Poisson algebra on the state space associated with the embedding labeled by $(n, 0)$ is

$$
\begin{aligned}
& \left\{A_{\mu}^{n}(\underline{x}), A_{\nu}^{n}\left(\underline{x}^{\prime}\right)\right\}=0 \\
& \left\{E_{n}^{\mu}(\underline{x}), E_{n}^{\nu}\left(\underline{x}^{\prime}\right)\right\}=0 \\
& \left\{A_{\mu}^{n}(\underline{x}), E_{n}^{\nu}\left(\underline{x}^{\prime}\right)\right\}=\delta_{\mu}^{\nu} \delta_{n}^{(3)}\left(\underline{x}-\underline{x}^{\prime}\right)
\end{aligned}
$$

The canonical momenta are computed from the Lagrangian;

$$
E_{n}^{t}(\underline{x})=0,{ }^{n} E^{\mu}(\underline{x})={ }^{n} P_{\rho}^{\mu} n_{\nu} F^{\nu \rho}(\underline{x})
$$

where we again use the decomposition into time-like and space-like parts. The canonical Hamiltonian is

$$
H_{n}=\int_{\Sigma_{n}} d \theta_{\underline{x}}\left[\frac{1}{2}{ }^{n} E^{\mu n} E_{\mu}-\frac{1}{4}^{n} F_{\mu \nu}^{n} F^{\mu \nu}-A_{t}^{n n} \partial_{\mu}{ }^{n} \pi^{\mu}\right]
$$


The equation of motion for $E_{n}^{t}$ implies the secondary constraint

$$
{ }^{n} \partial_{\mu}{ }^{n} E^{\mu}(\underline{x})=0
$$

which can be recognised as Gauss' law. The constraints form a first class pair. The corresponding gauge freedom is manifested in the fact that the equations of motion do not determine $A_{t}^{n}$ or $A_{L}^{n}$ where $A_{L}^{n}$ is the longitudinal part of $A^{n}$. The pull back of the symplectic two-form on $P_{n}$ to the constraint surface $C_{n}$ is degenerate because of the first class nature of the constraints. The reduced state space is obtained after gauge fixing and contains only four of the original eight degrees of freedom.

\subsection{History theory}

We follow the same procedure as before and consider sections of the trivial vector bundle $P \times \mathbb{R} \rightarrow \mathbb{R}$. So a history is a map

$$
t \mapsto\left(A_{\mu}^{n}(t ; x), E_{n}^{\nu}(t ; x)\right)
$$

and again, there is a unique pair $\left(A_{\mu}^{n}(X), E_{n}^{\nu}(X)\right)$ corresponding to such a history so we fix a $n$ and identify $\Pi_{n}$ with the abstract space $\Lambda^{1}(\mathcal{M}) \times \mathcal{X}(\mathcal{M})$. These history fields satisfy the covariant looking algebra,

$$
\begin{aligned}
& \left\{A_{\mu}^{n}(X), A_{\nu}^{n}\left(X^{\prime}\right)\right\}=0 \\
& \left\{E_{n}^{\mu}(X), E_{n}^{\nu}\left(X^{\prime}\right)\right\}=0 \\
& \left\{A_{\mu}^{n}(X), E_{n}^{\nu}\left(X^{\prime}\right)\right\}=\delta_{\nu}^{\mu} \delta^{(4)}\left(X-X^{\prime}\right)
\end{aligned}
$$

and the Louville, Hamiltonian and action operators are defined as

$$
\begin{aligned}
V_{n} & :=\int d^{4} X\left[E_{n}^{t} \partial_{n}^{t} A_{t}^{n}+{ }^{n} E^{\mu} \partial_{n}^{t n} A_{\mu}\right] \\
H_{n} & :=\int d^{4} X\left[{ }^{n} E_{\mu}{ }^{n} E^{\mu}+{ }^{n} F_{\mu \nu}{ }^{n} F^{\mu \nu}-A_{t}^{n n} \partial^{\mu n} E_{\mu}\right] \\
S_{n} & :=V_{n}-H_{n}
\end{aligned}
$$

At this point we note that, unlike in the case of the scalar field, the foliation dependence of these fields is empirically verified. It is a well-known fact that observations of magnetic and electric fields do depend on the state of motion of the observer. 


\subsection{Constraints}

We follow the procedure detailed for the vector field and work in the extended history space $\tilde{\Pi}_{n} \subset \Lambda^{1}(\mathcal{N}) \times \mathcal{X}(\mathcal{N})$. The extended history fields are written in the form

$$
\tilde{A}^{n}=\tilde{A}_{M}^{n}(X, s) d x^{M}, \tilde{E}_{n}=\tilde{E}_{n}^{M}(X, s) \partial_{M}
$$

and using a basis we have the decomposition of $\tilde{A}^{n}$ into time-like and spacelike components $\left(\tilde{A}_{s}^{n}, \tilde{A}_{t}^{n}, \tilde{A}_{i}^{n}\right)$. The action of the Poincare group is very similar to the corresponding definitions for the vector field. From now on we fix an $n$, and work in coordinates adapted to $n$, dropping the $n$-label for typographical convenience. The new feature of electromagnetism is, of course, gauge invariance. We extend the Louville, Hamiltonian and action functionals to the extended history space as

$$
\begin{aligned}
\tilde{V} & :=\int_{\mathcal{M}^{(e)}} d^{4} X\left[\tilde{E}^{s} \partial_{t} \tilde{A}_{s}+\tilde{E}^{t} \partial_{t} \tilde{A}_{t}+\tilde{E}^{i} \partial_{t} \tilde{A}_{i}\right] \\
\tilde{H} & :=\int_{\mathcal{M}^{(e)}} d^{4} X\left[\frac{1}{2} \tilde{E}_{i} \tilde{E}^{i}+\frac{1}{4} \tilde{F}_{i j} \tilde{F}^{i j}-\tilde{A}_{t} \partial^{i} \tilde{E}_{i}\right] \\
\tilde{S} & :=\tilde{V}-\tilde{H}
\end{aligned}
$$

As before we regard the equations

$$
\tilde{E}^{s}(X)=0, \tilde{E}^{t}(X)=0
$$

as the primary constraints of the theory. The corresponding secondary constraints follow from the Hamilitionian evolution of $\tilde{E}^{s}$ and $\tilde{E}^{t}$. The equation $\left\{\tilde{H}, \tilde{E}^{s}(X)\right\}=0$ is identically satisfied and $\left\{\tilde{H}, \tilde{E}^{t}(X)\right\}=0$ implies Gauss' law,

$$
\partial_{i} \tilde{E}^{i}(X)=0
$$

Gauss' law is conserved in internal time, $\left\{\tilde{H}, \partial_{i} \tilde{E}^{i}(X)\right\}=0$, as a consequence of the anti-symmetry of $\tilde{F}_{i j}$. The equations (116) and (117) are the first class constraints of the theory.

\subsection{External local symmetries}

To investigate the external local symmetries we define the extended action [13.

$$
\tilde{S}^{E}:=\tilde{S}-\int_{\mathcal{M}^{(e)}} d^{4} X\left[\lambda_{0} \tilde{E}^{s}+\lambda_{1} \tilde{E}^{t}+\lambda_{2} \partial_{i} \tilde{E}^{i}\right]
$$


The transformations

$$
\begin{aligned}
& \delta \tilde{A}_{s}(X)=\epsilon_{0}(X) \\
& \delta \tilde{A}_{t}(X)=\epsilon_{1}(X) \\
& \delta \tilde{A}_{i}(X)=\partial_{i} \epsilon_{2}(X)
\end{aligned}
$$

are generated by the functional

$$
\psi=\int_{\mathcal{M}^{(e)}} d^{4} X\left[\epsilon_{0} \tilde{E}^{s}+\epsilon_{1} \tilde{E}^{t}+\epsilon_{2} \partial_{i} \tilde{E}^{i}\right]
$$

The extended action is invariant under these transformations if

$$
\begin{aligned}
& \delta \lambda_{0}(X)=\partial^{t} \epsilon_{0}(X) \\
& \delta \lambda_{1}(X)=\partial^{t} \epsilon_{1}(X) \\
& \delta \lambda_{2}(X)=\epsilon_{1}(X)-\partial^{t} \epsilon_{2}(X)
\end{aligned}
$$

The symmetry of the total action, and therefore of the underlying Lagrangian theory is found by setting $\lambda_{2}=0$ (and $\delta \lambda_{2}=0$ ), thus eliminating the secondary constraint. The resulting transformations are

$$
\begin{aligned}
& \delta \tilde{A}_{s}(X)=\epsilon_{0}(X) \\
& \delta \tilde{A}_{\mu}(X)=\partial_{\mu} \epsilon_{2}(X)
\end{aligned}
$$

where $\mu=t, 1,2,3$. These transformations contain two arbitrary real functions on external space-time. Setting $\epsilon_{0}=0$ we obtain 'external' $U(1)$ gauge transformations:

$$
\delta \tilde{A}_{\mu}(X)=\partial_{\mu} \epsilon_{2}(X)
$$

which correspond to the symmetries of the external Maxwell tensor $\tilde{F}_{\mu \nu}=$ $\partial_{[\mu} \tilde{A}_{\nu]}$.

\subsection{Internal local symmetries}

The map $\tilde{A}_{M}(X) \mapsto \tilde{A}_{M}(X, s)$ is one-to-many for gauge systems. This introduces an extra ambiguity into the theory which is not contained in equations (126) and (127). We make this extra ambiguity explicit by introducing Lagrange multipliers to make the map $\tilde{A}_{M}(X) \mapsto \tilde{A}_{M}(X, s)$ one-to-one. To accomplish this we define the extended Hamiltonian;

$$
\tilde{H}_{s}^{E}\left[\lambda_{0}^{s}, \lambda_{1}^{s}, \lambda_{2}^{s}\right]:=\tilde{H}-\int_{\mathcal{M}_{s}^{(e)}} d^{4} X\left[\lambda_{0}^{s} \tilde{E}^{s}+\lambda_{1}^{s} \tilde{E}^{t}+\lambda_{2}^{s} \partial_{i} \tilde{E}^{i}\right]
$$


At each moment of internal time $s$, the integral is over $\mathcal{M}_{s}^{(e)}$ (the surface $s=$ const), and the Lagrange multipliers are arbitrary real valued functions $\lambda^{s}$ : $\mathcal{M}_{s}^{(e)} \rightarrow \mathbb{R}$. The extended Hamiltonian generates canonical transformations of the potential field

$$
\partial_{s} \tilde{A}_{M}(X, s)=\left\{H_{s}^{E}, \tilde{A}_{M}(X, s)\right\}
$$

and the map $\tilde{A}_{M}(X) \mapsto \tilde{A}_{M}(X, s)$ is given by the flow of the time-dependent vector field generated by $H_{s}^{E}$. Thus $\tilde{A}_{M}(X, s)$ is the solution of the following integral equation

$$
\tilde{A}_{M}(X, s)=\tilde{A}_{M}(X, 0)+\exp \left(\int_{0}^{s} d s^{\prime}\left\{\tilde{H}_{s^{\prime}}^{E}, \tilde{A}_{M}\left(X, s^{\prime}\right)\right\}\right)
$$

Because the transformation $\tilde{A}_{M}(X) \mapsto \tilde{A}_{M}(X, s)$ is canonical, it preserves the Poisson bracket so

$$
\left\{\tilde{A}_{M}(X, s), \tilde{E}^{N}\left(X^{\prime}, s\right)\right\}=\delta_{M}^{N} \delta^{(4)}\left(X-X^{\prime}\right)
$$

Gauge-equivalent histories correspond to different choices of the Lagrange multipliers in the extended Hamiltonian. The functional defined by

$$
\psi_{s}=\int_{\mathcal{M}_{s}^{(e)}} d^{4} X\left[\epsilon_{0}^{s} \tilde{E}^{s}+\epsilon_{1}^{s} \tilde{E}^{t}+\epsilon_{2}^{s} \partial_{i} \tilde{E}^{i}\right]
$$

generate transformations on $\mathcal{M}_{s}^{(e)}$ as follows:

$$
\delta \tilde{A}_{M}(X, s)=\left\{\psi_{s}, \tilde{A}_{M}(X, s)\right\}
$$

and these transformations take the form

$$
\begin{aligned}
& \delta \tilde{A}_{s}(X, s)=\epsilon_{0}^{s}(X) \\
& \delta \tilde{A}_{t}(X, s)=\epsilon_{1}^{s}(X) \\
& \delta \tilde{A}_{i}(X, s)=\partial_{i} \epsilon_{2}^{s}(X)
\end{aligned}
$$

In order that equation (130) is preserved by these transformations up to a change in the Lagrange multipliers associated with the primary constraints, the transformations must satisfy

$$
\begin{aligned}
& \delta \tilde{A}_{s}(X, s)=\epsilon_{0}^{s}(X) \\
& \delta \tilde{A}_{t}(X, s)=\partial_{s} \epsilon_{2}^{s}(X) \\
& \delta \tilde{A}_{i}(X, s)=\partial_{i} \epsilon_{2}^{s}(X)
\end{aligned}
$$


These are the internal local symmetry transformations of histories electromagnetism. They contain two arbitrary real valued functions on extended space-time, but are not just a trivial extension of the external local transformations to each moment of internal time. The transformation of $\tilde{A}_{t}$ contains a derivative with respect to internal time rather than external time. This is because the internal gauge transformations correspond to the symmetries of the internal field equations rather than the symmetries of the external field equations.

If we set $\epsilon_{0}=\partial^{s} \epsilon_{2}$ and restrict to a surface $t=$ const, we obtain 'internal' $U(1)$ gauge transformations:

$$
\delta \tilde{A}_{\bar{\mu}}(X, s)=\partial_{\bar{\mu}} \epsilon_{2}(X, s)
$$

where $\bar{\mu}$ runs over $1,2,3, s$. These are the symmetries of the internal Maxwell tensor $\tilde{F}_{\bar{\mu} \bar{\nu}}=\partial_{[\bar{\mu}} \tilde{A}_{\bar{\nu}]}$.

\subsection{Internal symmetries vs. external symmetries}

An arbitrary history satisfies the internal equations of motion. Therefore all histories that are related by internal gauge transformations should be regarded as physically equivalent. However, most histories will not satisfy the external equations of motion, and so need not respect the external symmetry transformations. Histories which are solutions to the external equations of motion are invariant under both internal and external symmetry transformations.

An internal local symmetry transformation is also an external local symmetry transformation if and only if

$$
\left(\partial^{t}-\partial^{s}\right) \epsilon_{2}^{s}(X)=0
$$

so the equation of motion $\left(\partial^{t}-\partial^{s}\right) \tilde{A}_{\mu}=0$ is conserved by these transformations. It is interesting to note that if equation (142) holds and we set $\epsilon_{0}=\partial^{s} \epsilon_{2}$ then we obtain $U(1)$ gauge transformations on $\mathcal{N}$ :

$$
\delta \tilde{A}_{M}(X, s)=\partial_{M} \epsilon_{2}^{s}(X)
$$

which are the symmetries of the five-dimensional Maxwell tensor $\tilde{F}_{M N}=$ $\partial_{[M} \tilde{A}_{N]}$. 


\section{Summary and Conclusion}

We have shown that the global symmetry transformations of geometric objects in a history theory suggests the introduction of an extra pair of fields. The extended history fields can then be interpreted as fields on $\mathcal{N}=\mathcal{M} \times \mathbb{R}$ that are covariant under the action of two Poincare groups, but not the $S O(2,3)$ group associated with $\mathcal{N}$. The history fields can be decomposed into two $S O(1,3)$-vectors at each point in the extended space-time, where the spatial components of these two vectors are the same.

In the case of the massive vector field, the theory contains two pairs of second class constraints, and in the case of electromagnetism, we obtain three first class constraints. In both these examples, the extra degrees of freedom can be set to zero, and eliminated from the theory by taking Dirac brackets. It seems reasonable to expect this pattern to continue in the extension to other constrained field theories as there is no physical information in the extra degrees of freedom. So although Lorentz covariance suggests that the fields should have five components, the extra constraints allow the extra variables to be eliminated.

In conclusion, the geometry of classical history theories is not fully understood. In the state space approach the solutions to the field equations are sections of tensor bundles associated to an $S O(1,3)$ principal bundle over $\mathcal{M}$. This formulation elegantly characterises the way that the fields transform under Lorentz transformations. If the history fields were covariant under $S O(2,3)$ then we would have a description of history fields in terms of sections of bundles associated to an $S O(2,3)$ principal bundle over $\mathcal{N}$. However the reality of the situation appears to be more complicated. There is an $S O(1,3)$ group associated to each surface of constant $t$, and to each surface of constant $s$, but the action of these two groups is intertwined in a non-trivial way. Similar remarks apply to the local symmetries in a history theory: they cannot be interpreted as the transformations of a $U(1)$ connection on a principal bundle over $\mathcal{N}$.

In a subsequent paper we will discuss the quantisation of histories electromagnetism using the BRST formalism. 


\section{Acknowledgements}

I would like to thank Chris Isham for his constant encouragement and support, and PPARC for a studentship.

\section{References}

[1] C.J. Isham. Quantum Logic and the histories approach to quantum theory. J. Math. Phys. 35 : 2157. 1994. gr-qc/9308006.

[2] C.J. Isham and N. Linden. Continuous histories and the history group in generalised quantum theory. J. Math. Phys. 36 : 5392. 1995. grqc/9503063.

[3] C.J. Isham, N. Linden, K. Savvidou and S. Schreckenberg. Continuous Time and Consistent Histories. J. Math. Phys. 37 : 2261. 1998. quantph/9711031.

[4] C.J. Isham and K. Savvidou. Quantising the foliation vector in Histories quantum field theory. In preparation

[5] K. Savvidou Continuous time in consistent histories (PhD thesis) $\mathrm{gr}$ qc/9912076

[6] K. Savvidou The action operator in continuous-time histories. J. Math. Phys. 40: 5657, (1999).

[7] K. Savvidou Poincare invariance for continuous-time histories. grqc/0104053

[8] K. Savvidou General Relativity histories theory. gr-qc/0104081

[9] C. Anastopoulos. Quantum Fields in Nonstatic background: a Histories Perspective. J. Math. Phys. 41 : 617. 2000. gr-qc/9903026.

[10] D. Noltingk A consistent histories approach to the Unruh effect. Int. J. Theo. Phys. 08 gr-qc/0005063

[11] S.J. Chang. Introduction to quantum field theory. Lecture Notes in Physics - Vol. 29, World Scientific, 1990. 
[12] D.M. Gitman and I.V. Tyutin. Quantization of fields with constraints, Berlin: Springer, 1990.

[13] M. Henneaux and C. Teitelboim. Quantization of gauge systems, Princeton University Press, 1992. 